\title{
Ganoderma lucidum on the inhibition of colorectal cancer
}

\author{
Hong Ye* and Alfred Bennett Jenson \\ James G. Brown Cancer Center, University of Louisville, Louisville, USA
}

\begin{abstract}
Ganoderma lucidum is used in East Asian countries for treatment of a variety of diseases such as hypertension, cardiovascular disease, hepatopathy, chronic hepatitis and many cancers. Accumulating evidence has shown that Ganoderma lucidum has significant benefit for the prevention and treatment of different cancers, particularly colorectal cancer. This review summarizes current Ganoderma lucidum research on colorectal cancer in clinical patient studies, animal model for examination, and molecular mechanistic investigations.
\end{abstract}

\section{Introduction}

Colorectal cancer is the fourth most common cancer worldwide and causes 50,000 deaths every year in the United States. About 1.2 million people in the US have been previously diagnosed, and approximately 150,000 new cases are diagnosed each year [1-3]. Treatment for colorectal patients depends on tumor location and diagnosis stages. Surgery is the most common treatment in early stage. Chemotherapy treatment, either alone or in combination with radiation therapy, is often given to patients at late stages regardless of previous surgery [1]. The chemotherapy agent 5-fluorical was first approved as colon cancer treatment in 1995. Since then, there have been more options for systematic treatment. However, large number of patients still have recurrence after being treated for either the local or the advanced colorectal cancer. Therefore, finding alternative approaches are becoming more important for colorectal cancer patients $[1,4]$.

Ganoderma lucidum has been widely used in China, Japan and other East Asian countries for more than 2000 years. It is also known as "Reishi" in Japan and "Ling Zhi" in China. The medicinal usage of Ganodema lucidum includes promotion of vitality, debility, hypertension, cardiovascular disease, bronchitis, arthritis [5-8]. Among these medicinal functions, the antitumor activity of Ganoderma lucidum has attracted special attention for its ability to inhibiting cellcycle arrest, inducing apoptosis and anti-mutagenesis [9-11].

This review provides an overview of Ganoderma lucidum on its role in colorectal cancer prevention and treatment. The primary focus of this review is on the observation of Ganoderma lucidum whole extract inhibiting colorectal cancer. We also draw upon several studies on colorectal cell lines including HCT116 cells and HT-29 cells. In the study of molecular mechanism of how Ganoderma lucidum effects colorectal cancer, identifying and extracting the active ingredient is essential. Therefore, this review also includes the introduction of major Ganderma lucidum active ingredients, and presents studies which demonstrate the effect of purified ingredients on cancer prevention responses, in particular polysaccharides and lanostainetype triterpentes.

Some studies of colorectal cancer treatment were performed using a combination of Ganoderma lucidum and other nature products. Due to the complexities of mixed herbs, these types of studies will not be reviewed here.

\section{The active ingredients in Ganoderma lucidum}

In the process of searching for active ingredients with anti-tumor activity from Ganoderma lucidum, two major groups of compounds were identified by several research laboratories. One group of compounds is polysaccharide (GLP), which mainly contains glucan and glyprotein. The other group is lanostaine-type triterpene, which mainly contains ganoderic acid, ganoderic alcohol and their derivate $[7,12,13]$. The primary activity of polysaccharide is to activate macrophage, neutrophil, dendritic cell, natural killer cell and lymphocytes [14-16]. The triterpene of Ganoderma lucidum is able to inhibit activities of farnesyl protein transferase and $5 a$-reductase $[17,18]$. Besides these two major compounds, sterol, lectin and some proteins have been shown to contribute to the medicinal applications [19-22].

Polysaccharide was extracted from Ganoderma lucidum with molecule weights ranging from $10 \mathrm{kDa}$ to more than $20 \mathrm{kDa}$ [23]. Polysaccharide plays a crucial role in Ganoderma lucidum bioactivities of antioxidants and antitumorogenesis. It also improves the immune response of the host, enhance the host's defense system, and stimulate interleukin-1 beta, TNFalpha, IL-6 of macrophage and interferongama (IFN-y) from T-lymphpcytes [24,25].

The first triterpenoid ganoderic acid A was reported 30 years ago. Since then, over 150 triterpenes were isolated including Ganoderic acid D, Ganoderic acid T, Ganoderic acid DM, and Ganodermanontirol [26]. Scientists have elucidated the orchestration of triterpenoid biological process in many publications [27]. Although the number of new triterpenoid identification is still growing, much attention has been focused on the pharmacological effects of medicinal fractions.

\section{The specificity and treatment of colorectal cancer}

This review is about colorectal cancer treatment by Ganoderma lucidum. We will first review the specificity for colorectal cancer to

Correspondence to: Hong Ye, James G. Brown Cancer Center, University of Louisville, 505 South Hancock Street, Louisville, KY, 40202, USA, Tel: 1-5028524047; E-mail: hong.ye@louisville.edu

Key words: ganoderma lucidum, cancer, colorectal cancer, nature product

Received: April 25, 2017; Accepted: May 08, 2017; Published: May 10, 2017 
understand the fundamental mechanism of how colorectal cancer originates. We will also review the available drugs and different treatments in each stage of colorectal cancer.

Colorectal cancer, one of the leading cancers in developed countries, cause up to 50,000 deaths every year in the United States. Both genetic and diet factors play essential roles in its occurrence and progression. Colorectal tumorigenesis progresses through several stages from initiation to progression. The primary genetic mutation leading to colorectal cancer is the APC pathway, which contributes to approximately $95 \%$ of colorectal cancer [28]. Loss of APC function leads to mutated epithelia cell expansion, giving rise to a small adenoma. Several other pathways, including RAS/RAF pathway, p53 pathway, drives tumor progression towards malignancy and metastasis [29].

Colorectal cancer initially starts from one or more generically mutated cells. The mutation takes many years to evolve. In the process of evolving, the development of colon cancer is highly affected by different dietary factors which contact epithelial cells in colorectum. Dietary components have ability to modify the development of this neoplastic process. Some diet, such as fat, are believed to have an important role in colorectal cancer [30]. A high incidence of colorectal cancer is almost always reported in populations of a developed countries' diet, such as highly animal meat consumption, while vegetable and fiber diet have the inverse result, due to their anticarcinogen functions, such as inducing of detoxifying enzymes by folate and antioxidants, binding to the luminal carcinogens, and fiber fermentation to produce protective volatile fatty acids.

For different stages of colorectal cancer, the treatments are different. At stage 0 , colorectal cancer is found only in the innermost lining of the colon. Surgery, including polypectomy to remove the polyp or local excision through a colonoscopy is curative. At stage 1, tumor has grown through several layers of colon, but does not spread. Surgery to remove the polyps or section of colon that contain the polyps is the standard treatment. At stage 2, the tumor has grown into the muscularis propria, a deeper, thick layer of muscle. Metastases are not present. Surgery to remove the section of the colon containing the cancer and nearby lymph nodes is treatment of choice, although chemotherapy might be needed after surgery. At stage 3, the tumor has grown through the muscularis propria and spread to nearby lymph nodes. Surgery followed by adjuvant chemo is the standard treatment. Some patients might need radiation therapy but only if the cancerous process in contained within a small area. At stage 4 , cancer has normally metastasized to distant sites such as liver, lungs or other organs and tissues. Chemothrapy, in combination with surgery, and targeted therapy are needed [31].

Among the chemotherapy agents, the individual chemotherapy agents include 5-Flruorouracil, Capecitabine, Irinotecan, Ocaliplatin, Bevacizumab, Cetuximab, and Panitumumab. The first four agents are small molecular compounds and the last three are monoclonal antibodies. Their anticancer mechanisms differ from each other.

5-Fluorouracil is a nucleoside analog. It blocks the enzyme thymidylate synthase and prevents the synthesis of thymine nucleotides and DNA replication. Capecitabine is converted to 5-FU by thymidine phosphorylase at the tumor site. Irinotecan is a derivative of camptothecin, found in the Camptotheca acuminate tree from China. Irinotecan has strong activity to inhibit the nuclear enzyme topoisomerase I, and to prevent uncoiling of DNA during replication. Oxaliplatin is a platinum based drug which forms both inter-and intra-strand cross links in DNA, preventing DNA replication and transcription, causing cell death.

The antibody-based drugs have drawn more attention recently because of their high specification and decreased toxicity. Babvacizumab binds to vascular endothelial growth factor A (VEGF-A) and inhibits blood-vessel formation. Cetuximab is a chimeric (human/mouse) monoclonal antibody that binds to the extracellular domain of the epidermal growth factor receptor (EGFR) and blocks ligand-induced receptor signaling. Panitumumab is a human monoclonal antibody whose function is similar to Cetuximab, and binds EGFR.

\section{Clinical data of Ganoderma lucidum treatment on colorectal cancer}

Most of the clinical trials of Ganoderma lucidum treatment of colorectal cancer were published in non-English journals, which limits the result of the studies for review. Among the colorectal tumor treatment by Ganoderma lucidum, a clinical trial was performed in Japan and published in English to evaluate tumor preventive effects. Among 225 enrolled patients, 123 patients were enrolled in the Ganoderma lucidum treatment group and 102 patients were nontreatment control group. During the 12-month trial, the patients in the treatment group took the water-soluble extract from $1.5 \mathrm{~g}$ of Ganoderma lucidum mycelia per day. The patients from the control group came to follow-up colonoscopy at the 12th month. In the treatment group, only 96 patients completed the trial.

These results showed that the mean \pm SE of adenoma number in the control group increased to $0.66 \pm 0.10$, while in the treatment group it decreased to $-0.42 \pm 0.21$. The mean size of adenomas increased to $1.73 \pm 0.28$ in the control group and were decreased to $-1.40 \pm 0.64$ $\mathrm{mm}$ in size in the treatment group. The study also found out that the polypoid-type adenomas was increased in the control group, but decreased in the treatment group. The changes in the number of adenomas were decreased to $-1.40 \pm 0.64 \mathrm{~mm}$ in the treatment group, and were increased to $0.66 \pm 0.10 \mathrm{~mm}$ in the control group. Overall, the data suggested that Ganoderma lucidum has ability to suppress the development of colorectal adenomas [32].

\section{Animal studies of Ganoderma lucidum treatment on colorectal cancer}

The protective effects of Ganoderma lucidum against colon cancer development were studied in a mouse model by Watanabe's group. The mice were first injected with NN-dimethyhydrazine (DMH) for 10 weeks to induce colon carcinogenesis, then the mice were fed the water-soluble fraction of Ganoderma lucidum for 10 weeks. Aberrant foci of crypts were examined, which is one of the earliest changes seen in the colon that may lead to cancer, and often are used as the biological end points to evaluate modulation of colonic carcinogenesis. General examination of the mice showed the average body weight did not change significantly, however, kidney weights in the Ganoderma lucidum treatment group were significantly larger compared to the regular non-treatment group. The aberrant crypts foci numbers appeared to be decreased in the Ganoderma lucidum feeding group. There was no obvious difference in the total tumor number between two groups, but the mean size was substantially reduced in the Ganoderma lucidum feeding group [33].

Watanabe's group further investigated whether Ganoderma lucidum has the protective effect against aberrant crypt formation by azoxymethane and small intestinal damage induced by the anti-cancer 
drug 5-FU. In the study, rats were given subcutaneous injections of carcinogenic compound azoxymethane once a week for 3 weeks followed by anti-cancer drug 5-FU injected intra-peritoneally three times at 5-day intervals. Administration of azoxymethane led to a dramatic loss in body weight. Taking anti-cancer drug 5-FU led to more weight loss. The most severe weight loss group took azoxymethane + 5-FU + Ganoderma lucidum. This study reported that 5-FU decreased the formation of aberrant crypts foci induced by azoxymethane, and that combination with Ganoderma lucidum further decreased the number of aberrant crypts foci. In addition, examining the intestinal damage discovered that the group fed with Ganoderma lucidum showed significant recovery from small intestinal damage induced by azoxymethane and 5-FU treatment [34].

The major active components of Ganoderma lucidum to be characterized includes polysaccharide and lanostane-type triterpene. The triterpene extract, containing ganoderic and lucidenic acid, was found to have activity of inhibiting growth and metastasis of lung carcinoma and hepatoma $[35,36]$. Sliva's group studied the triterpene extract inhibition activity by mouse colon carcinogenesis that induced the food-borne carcinogen 2-Amino-1-methyl-6-phenylimidazol [4,5b] pyridine (PhIP) and inflammation dextran sodium sulfate (DSS). Tumor were induced by the treatment of mice with PhIP and DSS combination. The triterpene extract reduced the tumor incidence. The study also showed that the high dose of triterpene was required to reducing neoplastic index, which was used to evaluate the severity of neoplastic lesion. Triterpene extract was able to suppress the focal hyperplasia induced by combination of PhIP and DSS. Furthermore, the triterpend extract was able to suppress the aberrant crypt foci induction by a combination of PhIP and DSS. All suppression functions were enhanced by high dosages of triterpene extract. In conclusion, Sliva's work has confirmed that triterpene extract is able to suppress colon inflammation and prevent tumor incidence and tumor multiplicity [37].

\section{Cell model and molecular mechanism studies of Ganoderma lucidum extract on colon cancer}

One of the challenges for nature product research is that nature product is a mixture containing diverse ingredients and multiple active chemical compounds. To separate the ingredient, identify the active ingredient and analyze the function for each ingredient have proved to be a complicated process. To determine how Ganoderma lucidum inhibits colorectal cancer, we first review the studies on the whole Ganoderma lucidum extract to determine how it affected tumor cell growth. Afterward, we individually reviewed polysaccharide and triterpene effect on colorectal cancer.

A group led by Barbara Pence took ethanolic extract of Ganoderma lucidum and studied its induction of apoptosis and the inhibition of different cytokine expression in human colonic carcinoma cell line HT29. Interestingly, Ganoderma lucidum extract was non-cytotoxic up to a dose of $1000 \mu \mathrm{g} / \mathrm{ml}$. Additionally, the extract was able to decrease COX-2 expression, and other cytokines including IL-8, MIP, VEGF and PDGF. It was also able to induce NO production [38].

Ganoderma lucidum polysaccharide has been shown to inhibit different cancer cell types by inducing cell apoptosis. Xingyao Xiong's laboratory specifically took human colon cancer cells HCT-116 and studied cell proliferation, cell migration, lactate dehydrogenase levels, and intracellular free calcium levels. This study showed that polysaccharide inhibited viability of HTC-116 cells and delayed
HCT-116 cell migration. Interestingly, the apoptosis induction was first observed by cell surface morphology changes and confirmed by transmission electron microscopy. Furthermore, Xiong lab has found polysaccharide significantly enhanced caspase- 8 activity, and both caspase- 3 and Fas were up-regulated in HTC-116 cell. The result indicated polysaccharide induces apoptosis through caspase dependent pathways [23].

Sliva's laboratory did elegant work on mechanistic study of Ganoderma lucidum triterpene on mouse colon carcinogenesis. To test whether triterpene extract from Ganodema lucidum was able to suppress proliferation of HT-29 cells, HT-29 cells were implanted subcutaneously in nude mice. After the tumor size reached 20 to 30 $\mathrm{mm}^{3}$, buffered triterpene extract was injected intraperitoneally into mice daily for 23 days. Control mice were injected with buffer alone. The data revealed that the triterpene extract treatment significantly suppressed the HT-29 tumor growth. The inhibition activity was associated with cell cycle arrest at G0/G1 followed by induction of autophagy. Additionally, triterpene extract induced formation of autophagic vacuoles and upregulated the expression of Beclin-1 and LC-3 proteins in colon cancer cells and tumors in the xenograft model. While the autophagy was medicated through the inhibition of P38 MAPK, the triterpene extract was able to suppress phosphorylation of P38 MAPK [39]. The group has further shown that the triterpene extract was able to down regulate PHIP/DSS-dependent expression of cyclin D1, COX-2, CYP1A2 and CYP3A4 in colon tissue and affect activities of transcription regulator of metabolic enzymes in vitro and modulates expression of CYP1A2, 4 and 1 in vivo [37].

Ganodermanontriol, a purified lanostanoid triterpene from Ganoderma lucidum, is able to inhibit proliferation of HCT 116 and HT-29 colon cancer cell lines [40]. Interestingly, the inhibition activity for HT-29 cells is stronger than for HTC-116 cells. Moreover, studies have found that ganodermanontriol suppressed the expression of cyclin D1, and down regulated expression of cyclin-depend kinase Cdk4 and PCNA, but the expression of Cdk-2, p21 and cyclin E were not affected. Since cyclin D1 is one of the main target genes regulated by $\beta$-catenin in colon cancer cells, the study further investigated $\beta$-catenin/LEF/ Tcf activity in HCT-116 and HT-29 cells. The results showed that ganodermanontriol markedly induced expression of $\beta$-catenin in HT29 cells but suppressed in HTC-116 cells [40].

Purified ganoderic acid Y (GA-T) from Ganoderma lucidum is able to inhibit proliferation of HTC-116 cells in a dose-dependent manner, promote spontaneous HCT-cell aggregation, and inhibit the adhesion of HCT116 cells to Matrigel. GA-T also suppresses the migration capability of 95-D cells. GA-T treatment is able to decrease the amount of MMP9 protein, suppresses uPA protein expression, suppresses TNFa-induced iNOS protein expression, inhibits p65 and I $\mathrm{KB}$ degradation. All the data taken together suggests that GA-T inhibits NF-kB activity [41].

\section{Conclusion}

Although some studies have been done for Ganoderma lucidum on colorectal cancer from clinical examinations and mechanistic investigations, more research is needed to be performed. The clinical studies that were unable to use purified compounds made it difficult to evaluate the quality of tested material. Even more challenging is to evaluate the quantity of active ingredients. For the mechanistic studies, two major groups of active ingredients were identified, polysaccharide and triterpene. However, more questions needed to be answered, for example: what are their receptors? Which pathways are activated by them? How do the multiple active ingredients work in harmony 
to reach the best therapeutic result for inhibiting cancerogenesis? Nevertheless, these remaining questions point to the exciting research topics in the upcoming years.

\section{Acknowledgments}

We thank Cassy Dale Luckett for her help with the critical reading of the manuscript.

\section{Disclosure statement}

There is on conflict interest for this manuscript.

\section{References}

1. Siegel R, DeSantis C, Virgo K, Stein K, Mariotto A, et al. (2012) Cancer treatment and survivorship statistics, 2012. CA Cancer J Clin 62: 220-241.[Crossref]

2. Torre LA, Siegel RL, Ward EM, Jemal A (2016) Global Cancer Incidence and Mortality Rates and Trends--An Update. Cancer Epidemiol Biomarkers Prev 25: 16-27.

3. Siegel R, Desantis C, Jemal A (2014) Colorectal cancer statistics, 2014. CA Cancer J Clin 64: 104-117.[Crossref]

4. Segal NH, Saltz LB (2009) Evolving treatment of advanced colon cancer. Annu Rev Med 60: 207-219.[Crossref]

5. Lin ZB (2005) Cellular and molecular mechanisms of immuno-modulation by Ganoderma lucidum. J Pharmacol Sci99: 144-153.[Crossref]

6. Shiao MS (2003) Natural products of the medicinal fungus Ganoderma lucidum: occurrence, biological activities, andpharmacological functions. Chem Rec 3: 172-80.

7. Boh B (2013) Ganoderma lucidum: a potential for biotechnological production of anticancer and immunomodulatory drugs. Recent Pat Anticancer Drug Discov 8: 255-287. [Crossref]

8. Gao Y, Zhou S (2003) Cancer Prevention and Treatment by Ganoderma, a Mushroom with Medicinal Properties. Food Reviews International 19: 275-325

9. Hu H, Ahn NS, Yang X, Lee YS, Kang KS (2002) Ganoderma lucidum extract induces cell cycle arrest and apoptosis in MCF-7 human breast cancer cell. Int J Cancer 102: 250-253.[Crossref]

10. Sliva D, Labarrere C, Slivova V (2002) Ganoderma lucidum suppresses motility of highly invasive breast and prostate cancer cells. Biochem Biophys Res Commun298: 603-612.

11. Lakshmi B, Ajith TA, Sheena N (2003) Antiperoxidative, anti-inflammatory, and antimutagenic activities of ethanol extract of the mycelium of Ganoderma lucidum occurring in South India. TeratogCarcinog Mutagen Suppl 1: 85-97.

12. Gao Y1, Zhou S, Jiang W, Huang M, Dai X (2003) Effects of ganopoly (a Ganoderma lucidum polysaccharide extract) on the immune functions in advanced-stage cance patients. Immunol Invest 32: 201-215.[Crossref]

13. Weng CJ, Yen GC (2010) The in vitro and in vivo experimental evidences disclose the chemopreventive effects of Ganoderma lucidum on cancer invasion and metastasis. Clin Exp Metastasis 27: 361-369.

14. Zhu XL, Chen AF, Lin ZB (2007)Ganoderma lucidum polysaccharides enhance the function of immunological effector cells in immunosuppressed mice. J Ethnopharmacol 111: $219-226$.

15. Lin KI, Kao YY, Kuo HK (2006) Reishi polysaccharides induce immunoglobulin production through the TLR4/TLR2-mediated induction of transcription factor Blimp-1. J BiolChem281: 24111-24123

16. Hua KF, Hsu HY, Chao LK (2007)Ganoderma lucidum polysaccharides enhance CD14 endocytosis of LPS and promote TLR4 signal transduction of cytokine expression. $J$ Cell Physiol 212: 537-550.

17. Lee S, Park S, Oh JW, Yang C (1998) Natural inhibitors for protein prenyltransferase. Planta Med 64: 303-308.[Crossref]

18. Liu J, Kurashiki K, Shimizu K, Kondo R (2006) 5alpha-reductase inhibitory effect of triterpenoids isolated from Ganoderma lucidum. Biol Pharm Bull 29: 392-395. [Crossref]

19. Ferreira IC, Heleno SA, Reis FS (2015)Chemical features of Ganoderma polysaccharides with antioxidant, antitumor and antimicrobial activities. Phytochemistry 114: 38-55.

20. Paterson RR (2006) Ganoderma - a therapeutic fungal biofactory. Phytochemistry67: 1985-2001.[Crossref]
21. Zhang CR, Yang SP, Yue JM (2008) Sterols and triterpenoids from the spores of Ganoderma lucidum. Nat Prod Res22: 1137-1142.[Crossref]

22. Ngai PH, Ng TB (2004) A mushroom (Ganodermacapense) lectin with spectacular thermostability, potent mitogenic activity on splenocytes, and antiproliferative activity toward tumor cells. BiochemBiophys Res Commun 314: 988-993.

23. Liang Z, Guo YT, Yi YJ (2014)Ganoderma lucidum polysaccharides target a Fas caspase dependent pathway to induce apoptosis in human colon cancer cells. Asian Pac J Cancer Prev 15: 3981-3986.

24. Ma C, Guan SH, Yang M (2008) Differential protein expression in mouse splenic mononuclear cells treated with polysaccharides from spores of Ganoderma lucidum. Phytomedicine 15:268-276.

25. Habijanic J, Berovic M, Boh B, Plankl M, Wraber B (2015) Submerged cultivation of Ganoderma lucidum and the effects of its polysaccharides on the production of human cytokines TNF- $\alpha$, IL-12, IFN- $\gamma$, IL-2, IL-4, IL-10 and IL-17. $N$ Biotechnol 32: 85-95. [Crossref]

26. Cheng S, Sliva D (2015)Ganoderma lucidum for cancer treatment: we are close but still not there. Integr Cancer Ther 14: 249-257.

27. Liu DZ, Zhu YQ, Li XF, Shan WG, Gao PF (2014) New triterpenoids from the fruiting bodies of Ganoderma lucidum and their bioactivities. Chem Biodivers 11: 982-986. [Crossref]

28. Powell SM, Zilz N, Beazer-Barclay Y (1992) APC mutations occur early during colorectal tumorigenesis. Nature 359: 235-237.

29. Vogelstein B, Kinzler KW (2001) Achilles' heel of cancer? Nature 412: 865-866 [Crossref]

30. Lipkin M, Reddy B, Newmark H, Lamprecht SA (1999) Dietary factors in human colorectal cancer. Annu Rev Nutr 19: 545-586.[Crossref]

31. Neuss MN, Gilmore TR, Belderson KM, Billett AL, Conti-Kalchik T, et al. (2017) 2016 Updated American Society of Clinical Oncology/Oncology Nursing Society Chemotherapy Administration Safety Standards, Including Standards for Pediatric Oncology. OncolNurs Forum 44: 31-43.[Crossref]

32. Oka S, Tanaka S, Yoshida S (2010) A water-soluble extract from culture medium of Ganoderma lucidum mycelia suppresses the development of colorectal adenomas. Hiroshima J Med Sci 59: 1-6.

33. Lu H, Uesaka T, Katoh O (2001) Prevention of the development of preneoplastic lesions, aberrant crypt foci, by a water-soluble extract from cultured medium of Ganoderma lucidum (Rei-shi) mycelia in male F344 rats. Oncol Rep 8: 1341-1345.

34. Watanabe H, Kashimoto N, Ushijima M, Tamura K (2013) Effects of a water-soluble extract of Ganoderma lucidum mycelia on aberrant crypt foci induced by azoxymethane and small-intestinal injury by 5-FU in F344 rats. Med MolMorphol 46: 97-103.

35. Kimura Y, Taniguchi M, Baba K (2002) Antitumor and antimetastatic effects on liver of triterpenoid fractions of Ganoderma lucidum: mechanism of action and isolation of an active substance. Anticancer Res 22: 3309-3318.

36. Weng CJ, Chau CF, Yen GC (2009) Inhibitory effects of ganoderma lucidum on tumorigenesis and metastasis of human hepatoma cells in cells and animal models. $J$ Agric Food Chem 57: 5049-5057.

37. Sliva D, Loganathan J, Jiang J (2012) Mushroom Ganoderma lucidum prevents colitisassociated carcinogenesis in mice. PLoS One 7: 47873.

38. Hong KJ, Dunn DM, Shen CL, Pence BC (2004) Effects of Ganoderma lucidum on apoptotic and anti-inflammatory function in HT-29 human colonic carcinoma cells Phytother Res 18: 768-770.

39. Thyagarajan A, Jedinak A, Nguyen H (2010) Triterpenes from Ganoderma Lucidum induce autophagy in colon cancer through the inhibition of p38 mitogen-activated kinase (p38 MAPK). Nutr Cancer 62: 630-40.

40. Jedinak A, Thyagarajan-Sahu A, Jiang J, Sliva D (2011)Ganodermanontriol, a lanostanoid triterpene from Ganoderma lucidum, suppresses growth of colon cancer cells through ß-catenin signaling. Int J Oncol 38: 761-767.

41. Chen NH, Liu JW, Zhong JJ (2010) Ganoderic acid T inhibits tumor invasion in vitro and in vivo through inhibition of MMP expression. Pharmacol Rep 62: 150-163. [Crossref]

Copyright: (C2017 Ye H. This is an open-access article distributed under the terms of the Creative Commons Attribution License, which permits unrestricted use, distribution, and reproduction in any medium, provided the original author and source are credited. 